\title{
THE STUDY OF KINETIC COLLOIDAL PARTICLES IN MAGNETIC FLUIDS USING DIFFRACTION EFFECT ON AN OPTICAL GRATING CREATED BY INTERSECTING LASER BEAMS
}

In this paper a short characteristic of magnetic fluids and some of their applications are presented a simple one-dimensional model of creation of thermal and absorption grating is explained. The method of generation of optical grating by using the interference two intersecting coherent laser beams is presented. In conclusion some possibilities of utilisation optical created grating are also indicated.

\section{Introduction}

Magnetic fluids are colloidal suspensions of single domain ferromagnetic particles $\mathrm{Fe}$ or $\mathrm{Fe}_{3} \mathrm{O}_{4}$, or other particles dispersed in a carrier liquid as, for example, water, mineral oil, etc. The particles, whose diameter is placed in the region $(5 \div 20) \mathrm{nm}$, could not set down due to Brownian motion. These particles interacted via the long-range magnetic forces and short range Van der Waals forces. Mutual interaction of these particles causes formation of agglomerates and then their sedimentation, and the long time stabilisation of the colloidal solution is ensured by surfactant. The surfactant covers the particles and thus essentially suppresses interaction. As surfactant matter a detergent or alcohol and so on is usually used.

The first magnetic fluid was prepared in 1938 by Elmore [1], in order to see the magnetic domains in some ferromagnetic materials. The Papell [2] developed the magnetic fluids in 1965 in connection with the cosmic program of NASA. In this case, will the fluid being the part of the fuel made it possible to transport fuel into rocket engine in weightless state. The next authors developed the ultra - stabilised fluids with cobalt particles, with different carrier liquids and different surfactants. The magnetic saturation was higher than in the case of the $\mathrm{Fe}_{3} \mathrm{O}_{4}$ particles. It turned out that the magnetic fluids share the hydrodynamic and the magnetic properties. These are very interesting for fundamental physical research and some technical application too. Now, we remind that the magnetic fluid can be used as the seal of very speedy rotating shafts. In this case the magnetic fluid plays the role of the packing ring. The magnetic fluid can be used as a filling of space, in which the coil of the loudspeaker oscillates, this secures the axial alignment.

At present the magnetic fluids find their application in biomedicine when the medicaments are purposefully transported [3], in radio-diagnostic as of a contrast-medium [4], etc.

\section{Creation of thermal grating - one-dimensional model}

If the sample of fluid containing colloidal particles is illuminated by the light whose intensity is harmonically varied on the direction perpendicular to the direction of the propagation of the light beam the fluid will be heated harmonically due to light absorption.

It is possible to obtain the harmonic dependence of the intensity of the light, using two coherent intersecting laser beams (Fig. 1).

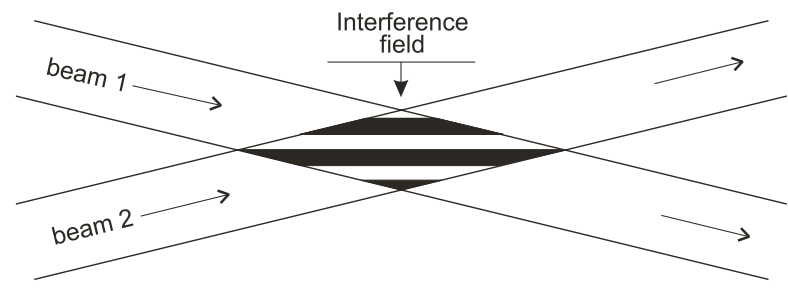

Fig. 1

When the sample of fluid is put into the interference field, the harmonic thermal field is created in the place where the beams are intersecting [5]. Harmonic variation of temperature will result in the thermal diffusion exchange of the colloidal particles between the places with different temperatures. This disturbs the equilibrium distribution of the particles (their concentration) and the diffusion due to concentration variations of particles starts between these places. After certain time the dynamic equilibrium takes place between these two processes and the periodical distribution of temperature and concentration of particles will be settled. Due to changes of the index of refraction the phase grating is created and due to the changes of concentration of particles the absorption grating is created too. The self-diffraction of light arises in this grating. When the interference field is switched off, the thermal

\footnotetext{
* Julius Štelina, Ctibor Musil

Department of Physics, University of Žilina, Velký diel, 01026 Žilina, Slovak Republic,
} 
optical grating vanishes after the relatively short time (of the order of few milliseconds), while the absorption grating exist essential a longer time. The destruction of the absorption grating can be observed through the decrease of diffracted beam intensity. This fact allows us to study the dynamics of the colloidal particles in the fluid.

The diffusion flux of particles in the created grating, in onedimensional case can be written

$$
\frac{\partial n}{\partial t}=-D \cdot \frac{\partial^{2} n}{\partial x^{2}}-S \cdot \frac{\partial^{2} \mathrm{~T}}{\partial x^{2}}
$$

In this equation $n$ is the concentration of particles, $t$ is time, $D$ means the diffusion constant of magnetic particles, $S$ is the thermal-diffusion coefficient (Soret constant), $T$ is the temperature and $x$ is the co-ordinate. In the case when the illumination is harmonic the equation (1) can be written as

$$
\frac{\partial n}{\partial t}=-D \cdot \frac{\partial^{2} n}{\partial x^{2}}-S \cdot D \cdot T_{0} \cdot n_{0} \cdot \Omega^{2} \cdot \sin (\Omega \cdot x)
$$

where $T_{0}$ is the amplitude of periodic modulation of the sample temperature, $n_{0}$ means density of the particles and $\Omega=\frac{2 \cdot \pi}{\Lambda}$ is the space frequency of the grating ( $\Lambda$ is the grating constant).

The solution of this equation is

$$
n(x, t)=n_{0} \cdot S \cdot T_{0} \cdot\left(1-\exp \left(-D \cdot \Omega^{2} \cdot t\right) \cdot \sin (\Omega \cdot x)\right.
$$

From this term we can see that the amplitude of the particle density in equilibrium is

$$
n_{s}=n_{0} \cdot S \cdot T_{0}
$$

We can also see from Eq. (3), that after the heating is switched off the concentration grating decreases exponentially with time constant

$$
\tau=\frac{1}{D \cdot \Omega^{2}}
$$

Using grating constant $\Lambda$ the diffusion constant $D$ can be expressed as

$$
D=\frac{\Lambda^{2}}{8 \cdot \pi^{2} \cdot \tau}
$$

From this we can see that $\tau$ is a quadratic function of the grating constant according to the expression

$$
\tau=\frac{1}{8 \cdot \pi^{2} \cdot D} \Lambda^{2}
$$

It is known that the diffusion coefficient is connected with mobility $\mu$ of the particles by Einstein's relation

$$
D=\mu \cdot k \cdot T,
$$

where $k=1.38 \cdot 10^{-23} \mathrm{~J} \cdot K^{-1}$ is the Boltzmann constant
In the first approximation we will assume that the particles are of spherical shape. Then the viscosity of the based fluid can be expressed according to the equation

$$
\eta=\frac{1}{6 \cdot \pi \cdot \mu \cdot r}=\frac{k \cdot T}{6 \cdot \pi \cdot D \cdot r},
$$

where $r$ is the effective radius of the colloidal particles. We use the Poisson relation which determines the connection between the force acting on the spherical particles and their mobility.

\section{Experiment and results}

The principal scheme of the set-up created in our department of physics is demonstrated in Fig. 2.

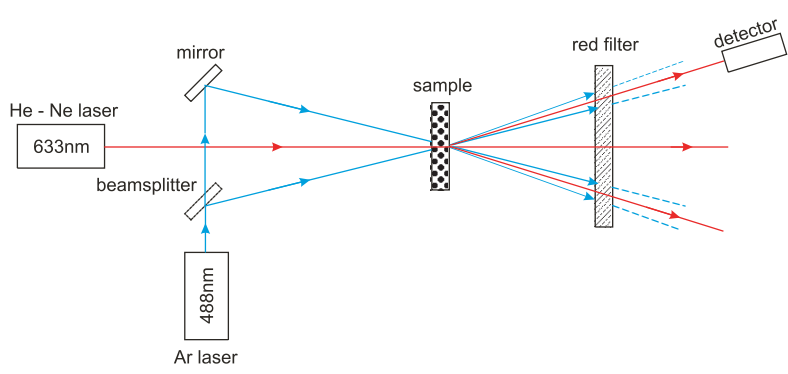

Fig. 2

The Ar laser beam ( $488 \mathrm{~nm}$ ) is divided with a beam-splitter into two coherent beams with the small trajectory difference. The sample of magnetic fluid, with thickness in the region $(60 \div 150) \mu \mathrm{m}$ was placed in coherent intersecting laser beams, where the interference field is present (Fig. 1). The interference field creates the thermal grating in the fluid due to absorption of the light. We can observe either self-diffraction or the optical diffraction of the $\mathrm{HeNe}$ (if we decide to use it). The disappearing of the diffraction grating can be seen on auxiliary reading $\mathrm{HeNe}$ beam that is detected and registered on PC.

The typical time dependence of creation and disappearance of grating in our magnetic fluid is presented in Fig. 3. The time dependence of disappearance of grating may be seen in Fig. 4 . This dependence has been evaluated and the time constant of the disappearance $\tau=18,2 \mathrm{~s}$ has been obtained. This time constant corresponds with the diffusion coefficient $D=1.2 \cdot 10^{-11} \mathrm{~m}^{2} \cdot \mathrm{s}^{-1}$, which is in good agreement with another authors [9], [12].

The quadratic dependence of time constant of disappearance on the grating constant for the first beam (Eq. (9)), has been experimentally verified and is presented in Fig. 5.

\section{Some applications}

Some technical applications of magnetic fluids were mentioned in the introduction of this paper. The optical grating created by 
two intersecting coherent laser beams in magnetic fluids could be used as an optical-electronic element, which has some coincident properties as can be seen in Fig. 6a,b.

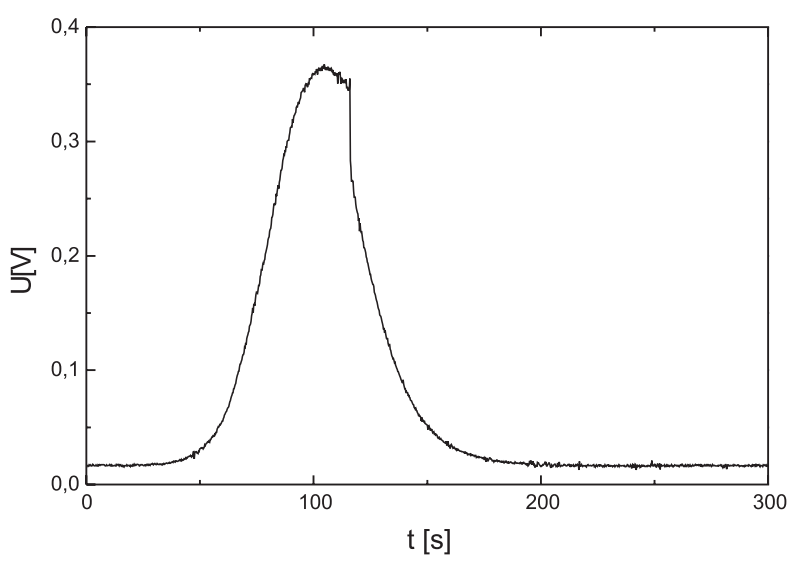

Fig. 3

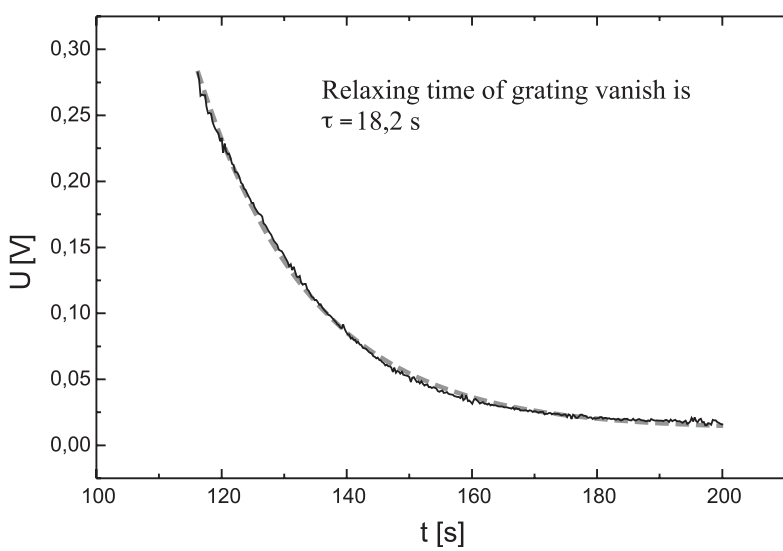

Fig. 4.

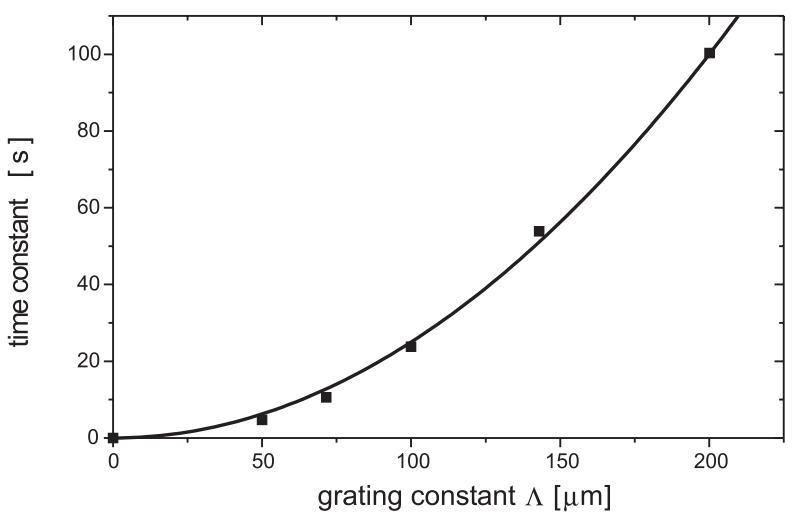

Fig. 5

The signals A, B come on optical grating, realised by magnetic fluid. The coherent signals A or B with wavelength $\lambda$ are detected on output side separately (Fig. 6a). If both beams appear at the same time, after interference in their intersecting place, the signal $\mathrm{C}$ will be also detected on the output.

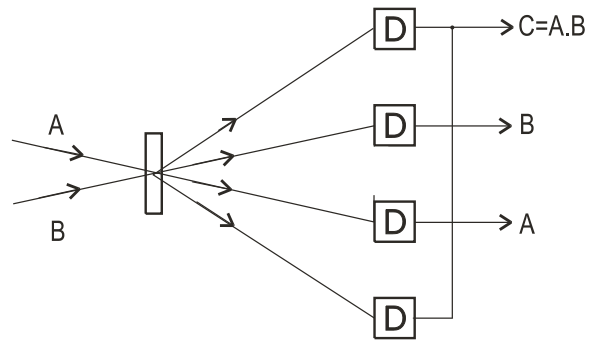

a)

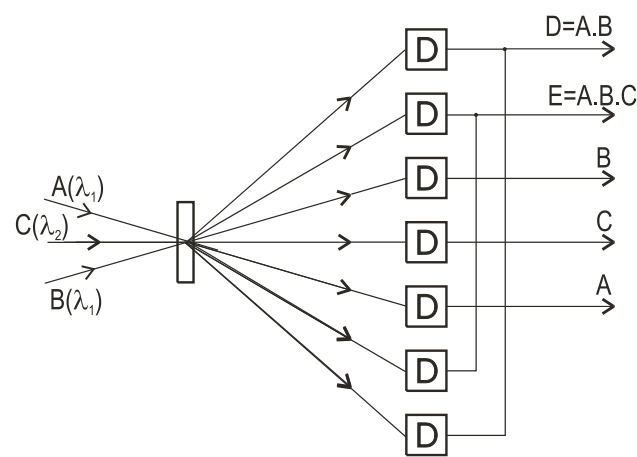

b)

Fig. $6 a, b$

In Fig. $6 \mathrm{~b}$ the case of three canal system is shown, in which coherent signals A and B work with wave-length $\lambda_{1}$. The laser beam $\mathrm{C}$ works on wave-length $\lambda_{2}$, for this the diffraction angle is different. The laser signals A, B or C can be detected on output side separately. The signal $\mathrm{D}$ can be detected if the interfering signals $\mathrm{A}$ and $\mathrm{B}$, which the optical grating create, are present in the same time, while signal $\mathrm{E}$ is detected if the signals $\mathrm{A}, \mathrm{B}$ and $\mathrm{C}$ are all presented at the same time.

The disadvantage of the presented optical-electronic elements is their slowly response, but in some cases this could turn into advantage, e.g. in cases where the time delay is useful.

\section{Conclusion}

From the preliminary results it can be seen that using the grating created by interference light field in the sample of the magnetic fluid and with the study of dynamic of its disintegration it is possible to obtain information about diffusion constant (Eq. (6)), the diameter of particles (Eq. (9)), if the viscosity of based fluid is known. Or vice versa the information about properties of the based medium, for example about its viscosity can be obtained, if the effective diameter is known (Eq. (9)). The possibility of other practical applications has been shown as well. 


\section{References}

[1] ELMORE, W. C.: Phys. Rev. 54 (1938), 309 and 1092

[2] PAPELL, S. S.: U.S. Patent, 3215572 (1965)

[3] RUUGE, E. K., RUSETSKI, A. N.,: J. Magn. Magn. Mater. 122 (1993), 335

[4] TSYB, A. F., AMOSOV, I. S., BERKOVSKY, B. M. a kol.: J. Magn. Magn. Mater. 39 (1983)

[5] SENDERÁKOVÁ, D., CSÓKOVÁ, Z., ŠTRBA, A.: A Contribution to Laser Induced Thermal Phase Gratings in Wave| and Quantum Aspects of Contemporary Optics, Jerzy Nowak, Marek Zajac, Editors, Proceedings of SPIE Vol. 3320, (1998), 217

[6] TIMKO, M., KOVÁČ, J., ZENTKO, A., KONERACKÁ, M., KOPČANSKY, P.: Magnetic fluids the properties and the possibilities of a technical using, Institute of the exp. phys. SAV, Košice

[7] EICHLER, H. J., GUNTER, P. and POHL, D. W.: Laser-Induced Dynamic Gratings, Springer- Verag Berlin, Heidelberg, 1986.

[8] MUSIL, C., ŠTELINA, J., TUREK, I.: The creating of diffraction grating in magnetic fluid using of Ar laser beam, The 10. conference of Slovak physicists, 10.-14.11.1997, Stará Lesná - Vysoké Tatry

[9] TUREK, I., ŠTELINA, J., MUSIL, C., TIMKO, M., KOPČANSKÝ, P., KONERACKÁ, M., TOMČO, L.: The effect of selfdiffraction in magnetic fluids, J. Magn. Magn. Mater. 201 (1999), 167

[10] KONPČANSKÝ, P., TOMČO, L., TIMKO, M., OCELÍK, V., KONERACKÁ, M., TUREK, I., ŠTELINA, J. AND MUSIL, C.: The Dynamics of the Soret Effect in Thin Film of Magnetic Fluid, Acta Physica Polonica A, No. 5, Vol. 97 (2000), p. 875 - 878

[11] TUREK, I., ŠTELINA, J., MUSIL, C., KOPČANSKÝ, P., TIMKO, M., KONERACKÁ, M., POTOČNÁ, J., JURÍKOVÁ, A., TOMČO, L.: Self-diffraction of the light in magnetic fluids, Czechoslovak Journal of Physics, Vol. 52 (2002), Suppl. A, 269

[12] MEZULIS, A., BLUMS, E., BOURDON, A., DEMOUCHY, G.: Thermodiffusion - induced optical index grating in ferrocolloids: Determination of transport coefficients, Fourth International PAMIR Conference on MHD at Dawn of Third Millenium, Presquile de Giens, France Sept. 18 - 22, 2000, p. 781 